\title{
Factorization for hadronic heavy quarkonium production *
}

\author{
Jian-Wei Qiu ${ }^{1}$ \\ Department of Physics and Astronomy, Iowa State University \\ Ames, Iowa 50011, U.S.A. \\ Physics Department, Brookhaven National Laboratory \\ Upton, New York 11973-5000, U.S.A.
}

\begin{abstract}
We briefly review several models of heavy quarkonium production in hadronic collisions, and discuss the status of QCD factorization for these production models.
\end{abstract}

Key words: Heavy quarkonium, factorization

PACS: 12.38.Bx, 12.39.St, 13.87.Fh, 14.40Gx

\section{Introduction}

RHIC has produced sufficient evidence that a new state of hot and dense matter of quarks and gluons, or the quark-gluon plasma (QGP), was formed in ultrarelativistic heavy ion collisions [1]. Anomalous suppression of heavy quarkonium, $\mathrm{J} / \psi$, was suggested as a good probe for discovering the QGP [2]. Because of the heavy mass, production of heavy quark pairs takes place at a very short time and is unlikely to interfere with the formation of QGP. On the other hand, the probability for the heavy quark pair to become a bound meson in the QGP could be suppressed due to the Debye screening from color charges in the medium.

However, the suppression of heavy quarkonia in a dense medium also depends on how the bound state was formed. A quicker formation would give less time for the medium's color charges to break the coherence of the heavy quark pair,

\footnotetext{
* Supported in part by the U.S. Department of Energy under Grant No. DE-FG0287ER40371 and Contract No. DE-AC02-98CH10886.

1 Email address: jwq@iastate.edu
}

Preprint submitted to Elsevier Science 20 October 2018 
and would require a higher color density and/or temperature to produce the suppression. It is therefore very important to ask how well we can calibrate the suppression in heavy ion collisions to extract useful information on medium properties. In this talk, I first briefly review several models for heavy quarkonium production, and then, discuss the status of QCD factorization for these production models, and finally, give a brief summary and outlook.

\section{Production models}

Heavy quarkonium production has been the subject of a vast theoretical literature and of intensive experimental study [3]. In order to produce a heavy quarkonium in hadronic collisions, the energy exchange in the collisions has to be larger than the invariant mass of the produced quark pair $\left(\geq 2 m_{Q}\right.$ with heavy quark mass $m_{Q}$ ). Since the binding energy of a heavy quarkonium of mass $M$ is much less than heavy quark mass, $\left(M^{2}-4 m_{Q}^{2}\right) / 4 m_{Q}^{2} \ll 1$, the transition from the pair to a meson is sensitive to soft physics. The quantum interference between the production of the heavy quark pairs and the transition process is powerly suppressed by the heavy quark mass, and the production rate for a heavy quarkonium state, $H$, up to corrections in powers of $1 / m_{Q}$, can be factorized as,

$$
\sigma_{A+B \rightarrow H+X} \approx \sum_{n} \int d \Gamma_{Q \bar{Q}} \sigma_{A+B \rightarrow Q \bar{Q}[n]+X}\left(\Gamma_{Q \bar{Q}}, m_{Q}\right) F_{Q \bar{Q}[n] \rightarrow H}\left(\Gamma_{Q \bar{Q}}\right)
$$

with a sum over possible $Q \bar{Q}[n]$ states and an integration over available $Q \bar{Q}$ phase space $d \Gamma_{Q \bar{Q}}$. The nonperturbative transition probability $F$ for a pair of off-shell heavy quark $(\psi)$ and antiquark $(\chi)$ to a quarkonium state $H$ is proportional to the Fourier transform of following matrix elements

$$
\sum_{N}\left\langle 0\left|\chi^{\dagger}\left(y_{1}\right) \mathcal{K}_{n} \psi\left(y_{2}\right)\right| H+N\right\rangle\left\langle H+N\left|\psi^{\dagger}\left(\tilde{y}_{2}\right) \mathcal{K}_{n}^{\prime} \chi\left(\tilde{y}_{1}\right)\right| 0\right\rangle
$$

where $y_{i}\left(\tilde{y}_{i}\right)$ are coordinates, and $\mathcal{K}_{n}$ and $\mathcal{K}_{n}^{\prime}$ are local combinations of color and spin matrices for the $Q \bar{Q}$ state $n$. A proper insertion of Wilson lines to make the operators in Eq. (2) gauge invariant is implicit [4]. In Eq. (2), $\sigma_{A+B \rightarrow Q \bar{Q}[n]+X}$, represents the production of a pair of on-shell heavy quarks and is calculable in perturbative QCD [5]. The debate on the production mechanism has being focused on the transition from the pair to the meson.

Color-singlet model The color-singlet model (CSM) assumes that only a color singlet heavy quark pair with the right quantum number can become a quarkonium of the same quantum number and the transition from the pair to a meson is given by the quarkonium wave function [6]. $\sigma_{A+B \rightarrow Q \bar{Q}+X}$ 's dependence on relative momentum of the pair is neglected, and $\int d \Gamma_{Q \bar{Q}} F_{Q \bar{Q} \rightarrow H}$ in Eq. (1) is 


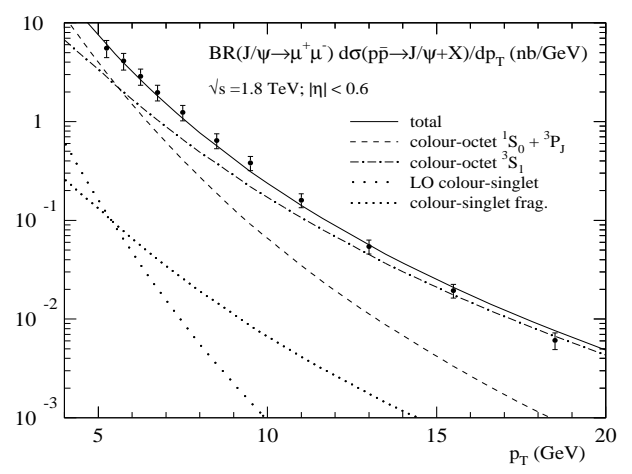

(a)

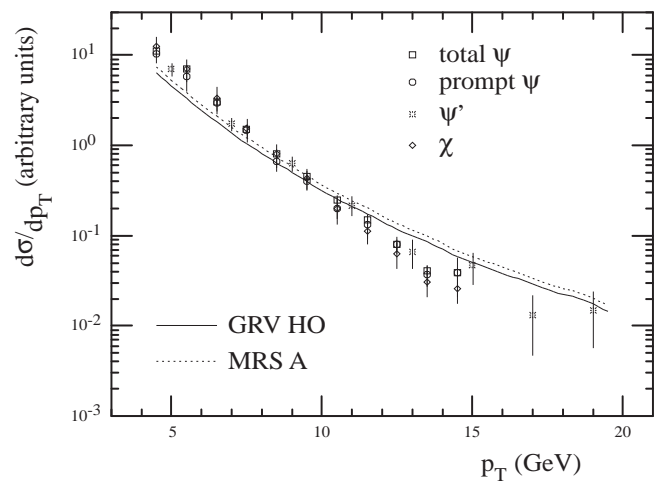

(b)

Fig. 1. Charmonium cross section as a function of $p_{T}$ along with the CDF data points [8] and the theory curves from NRQCD model from Ref. [7] (a), and CEM from Ref. [10] (b).

equal to the matrix element in Eq. (2) evaluated at $y_{i}\left(\tilde{y}_{i}\right)=0$, which is proportional to the square of coordinate-space quarkonium wave function at the origin, $\left|R_{H}(0)\right|^{2}[3]$, and therefore,

$$
\sigma_{A+B \rightarrow H+X}^{\mathrm{CSM}} \propto \sigma_{A+B \rightarrow Q \bar{Q}[H]+X}\left(m_{Q}\right)\left|R_{H}(0)\right|^{2} .
$$

The same wave function appears in both production and decay, and the model provides absolutely normalized predictions. It works well for $\mathrm{J} / \psi$ production in deep inelastic scattering, photon production, and some low energy experiments [3], but fails to predict the CDF data, see the dotted lines in Fig. 1(a) [7].

Color evaporation model The color evaporation model (CEM) assumes that all $Q \bar{Q}$ pairs with invariant mass less than the threshold of producing a pair of open-flavor heavy mesons, regardless their color, spin, and invariant mass, have the same probability to become a quarkonium [9]. That is, the $F_{Q \bar{Q}[n] \rightarrow H}$ in Eq. (1) is a constant for a given quarkonium state, $H$, and therefore,

$$
\sigma_{A+B \rightarrow H+X}^{\mathrm{CEM}} \approx f_{H} \int_{2 m_{Q}}^{2 M_{Q}} d m_{Q \bar{Q}} \sigma_{A+B \rightarrow Q \bar{Q}+X}\left(m_{Q \bar{Q}}\right)
$$

with open-flavor heavy meson mass $M_{Q}$ and a constant $f_{H}[9]$. With a proper choice of $m_{Q}$ and $f_{H}$, the model gives a reasonable description of almost all data including the CDF data, as seen in Fig. 1(b).

Nonrelativistic QCD model The Nonrelativistic QCD (NRQCD) model is based on the fact that the typical heavy quark rest-frame kinetic energy and binding energy, $m_{Q} v^{2}$, in a heavy quarkonium is much smaller than the heavy quark mass. The model separates the physics at scales of order $m_{Q}$ and higher from the dynamics of the binding by using NRQCD, an effective field theory [11]. It provides a systematic prescription to calculate the physics at $m_{Q}$ orderby-order in powers of $\alpha_{s}$, and expands the nonperturbative dynamics in terms 


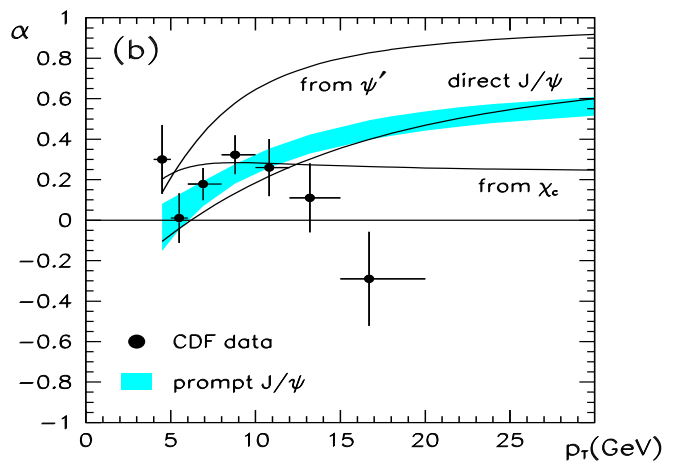

(a)

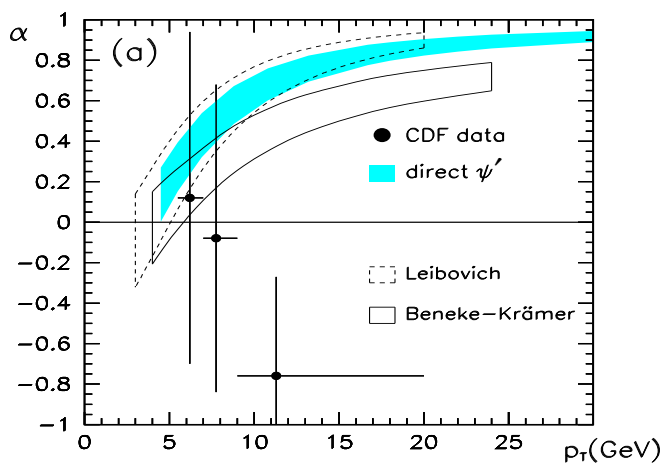

(b)

Fig. 2. From Ref. [7], NRQCD predictions of charmonium polarizations are compared with the CDF data [12].

of local matrix elements in power series of heavy quark velocity $v[7,11]$,

$$
\sigma_{A+B \rightarrow H+X}^{\mathrm{NRQCD}}=\sum_{n} \hat{\sigma}_{A+B \rightarrow Q \bar{Q}[n]+X}\left\langle\mathcal{O}_{n}^{H}\right\rangle
$$

where the $\mathcal{O}_{n}^{H}$ are NRQCD operators for the state $H$ [11],

$$
\mathcal{O}_{n}^{H}(0)=\chi^{\dagger}(0) \mathcal{K}_{n} \psi(0)\left(a_{H}^{\dagger} a_{H}\right) \psi^{\dagger}(0) \mathcal{K}_{n}^{\prime} \chi(0)
$$

where $a_{H}^{\dagger}$ is the creation operator for $H, \chi(\psi)$ are two component Dirac spinors, and $\mathcal{K}_{n}$ and $\mathcal{K}_{n}^{\prime}$ are defined in Eq. (2) and can also involve covariant derivatives. At higher orders in $v$, the operator $\mathcal{O}_{n}^{H}$ can have additional dependence on field strength as well as more fermion fields. The factorization in Eq. (5) could be understood from Eq. (1) by expanding the $\sigma_{A+B \rightarrow Q \bar{Q}[n]+X}$ at heavy quark relative momentum, $q=\left(p_{Q}-p_{\bar{Q}}\right) / 2=0$. The moments, $\int d \Gamma_{Q \bar{Q}} q^{N} F_{Q \bar{Q} \rightarrow H}$, lead to local matrix elements with high power of $v$.

The NRQCD model allows every $Q \bar{Q}[n]$ state to become a bound quarkonium, while the probability is determined by corresponding nonperturbative matrix elements $\left\langle\mathcal{O}_{n}^{H}\right\rangle$. Its octet contribution is the most important one for high $p_{T}$ quarkonium production at collider energies [7]. The NRQCD model has been most successful in interpreting data [3,7], as seen in Fig. 1(a).

Quarkonium polarization and other models The key difference between the NRQCD model and the CEM is the prediction on quarkonium polarization. Once the matrix elements $\left\langle\mathcal{O}_{n}^{H}\right\rangle$ are determined, the NRQCD model can systematically calculate the polarization of produced heavy quarkonia. We cannot calculate the polarization in CEM because of the uncontrolled radiation from the quark pair. The polarization of quarkonia at large $p_{T}$ was considered a definite test of the NRQCD model [7]. But, the model has failed the test, as seen in Fig. 2, if the CDF data hold [12]. Several improved and new models have been proposed to address the issues of polarization [13]. 


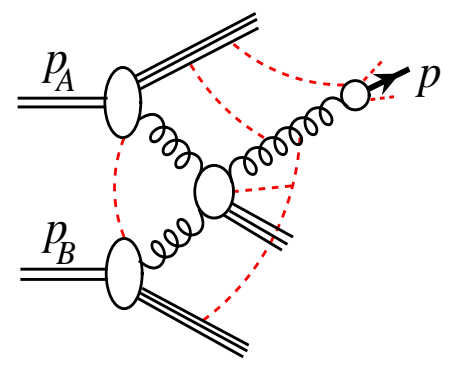

(a)

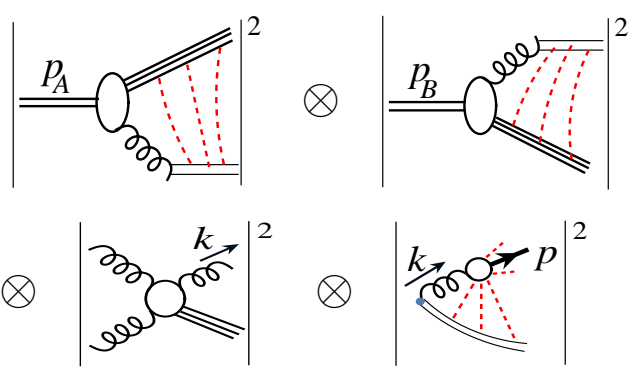

(b)

Fig. 3. Sketch for process $A+B \rightarrow H(p)+X$ : (a) sample scattering amplitude with possible factorization breaking soft interactions indicated by dashed lines, and (b) factorization with Wilson lines indicated by thin double lines.

\section{Factorization}

Heavy quarkonium production in hadronic collisions involves both perturbative and nonperturbative scales. Nonperturbative physics appears not only in the transition from the heavy quark pair to a bound state but also in incoming hadron wave functions. A typical scattering amplitude for quarkonium production, as sketched in Fig. 3(a), can have soft and nonperturbative interactions between incoming hadrons as well as between the spectators and the formation process. These soft interactions may introduce process dependence to the nonperturbative matrix elements, and consequently, spoil the predictive powers of Eqs. (4) and (5).

A proof of the factorization needs to: 1) show that the square of the scattering amplitude in Fig. 3(a), after summing over all amplitudes with the same initial and final states, can be expressed as a convolution of the probabilities, as sketched in Fig. 3(b); each probability represents a square of sub-amplitudes and is evaluated at its own momentum scale(s); 2) show that the piece evaluated at perturbative scale(s) is infrared safe and those evaluated at nonperturbative scales are universal.

As argued in Ref. [5], cross sections for producing on-shell heavy quark pairs can be computed in terms of QCD factorization. Therefore, the right-hand-side of the CEM formalism in Eq. (4) is perturbatively calculable for quarkonium production at a large $p_{T}$, and the $f_{H}$ should be universal within the model because soft interactions in Fig. 3(a) are suppressed by powers of $1 / p_{T}$. However, the factorization statement does not provide justification that the transition from a heavy quark pair to a quarkonium state is independent of the pair's invariant mass $m_{Q \bar{Q}}$, spin, and other quantum numbers. When $p_{T} \ll m_{Q}$, the universality of $f_{H}$ may not be valid because of the spectator interactions.

Fully convincing arguments have not yet been given for NRQCD factorization formalism in Eq. (5) [7,11]. Since the spectator interaction between the beam 
jet and the jet of heavy quark pair should be suppressed by powers of $1 / p_{T}$, one might expect the NRQCD factorization formalism to work at large $p_{T}$. When $p_{T} \gg m_{Q}$, heavy quarkonium production is similar to the single light hadron production, and is dominated by parton fragmentation. The cross section is proportional to the universal parton-to-hadron fragmentation functions [4],

$$
\sigma_{A+B \rightarrow H+X}\left(p_{T}\right)=\sum_{i} \hat{\sigma}_{A+B \rightarrow i+X}\left(p_{T} / z, \mu\right) \otimes D_{H / i}\left(z, m_{Q}, \mu\right)+\mathcal{O}\left(m_{H}^{2} / p_{T}^{2}\right) .
$$

Here, $\otimes$ represents a convolution in the momentum fraction $z$. The cross section $\hat{\sigma}_{A+B \rightarrow i+X}$ includes all information on the incoming state, including convolutions with parton distributions of hadrons $A$ and $B$ at factorization scale $\mu$, as sketched in Fig. 3(b). As a necessary condition for NRQCD factorization in Eq. (5), the following factorization relation,

$$
D_{H / i}\left(z, m_{Q}, \mu\right)=\sum_{n} d_{i \rightarrow Q \bar{Q}[n]}\left(z, \mu, m_{Q}\right)\left\langle\mathcal{O}_{n}^{H}\right\rangle,
$$

is required to be valid to all orders in $\alpha_{s}$ and all powers in $v$-expansion for all parton-to-quarkonium fragmentation functions [4]. In Eq. (8), $d_{i \rightarrow Q \bar{Q}[n]}$ describes the evolution of an off-shell parton into a heavy quark pair in state $[n]$, including logarithms of $\mu / m_{Q}$, and should be infrared safe [4].

The factorization relation in Eq. (8) was tested up to next-to-next-to-leading order (NNLO) in $\alpha_{s}$ at $v^{2}$ order in Ref. [4], as well as at finite $v$ in Ref. [14]. Consider representative NNLO contributions to the fragmentation process of transforming a color octet heavy quark pair to a singlet, as sketched in Fig. 4. The individual classes of diagrams in Fig. 4(I) and (II), for which two gluons are exchanged between the quarks and the Wilson line, satisfy the infrared cancellation conjecture of Ref. [11], by summing over the possible cuts and connections to quark and antiquark lines, as do diagrams that have three gluon-eikonal vertices on the quark pair and one on the Wilson line [4]. For Fig. 4(III) type of diagrams, however, with a three-gluon interaction, this cancellation fails. By summing over all contributions to second order in the relative momentum $q$, it was found that the fragmentation function has a noncanceling real pole [4]

$$
\mathcal{I}_{2}^{(8 \rightarrow 1)}(v)=-\alpha_{s}^{2} \frac{1}{3 \varepsilon} v^{2} .
$$

From Fig. 4(III), this infrared divergence is not topologically factorizable and can not be absorbed into the matrix elements $\left\langle\mathcal{O}_{n}^{H}\right\rangle$. As demonstrated in Ref. [4], as a necessary condition for restoring the NRQCD factorization at NNLO, the conventional $\mathcal{O}\left(v^{2}\right)$ octet NRQCD production matrix elements $\left\langle\mathcal{O}_{n}^{H}\right\rangle$ must be modified by incorporating Wilson lines that make them manifestly gauge invariant, so that the infrared divergence in Eq. (9) can be absorbed into the gauge-completed matrix elements. 


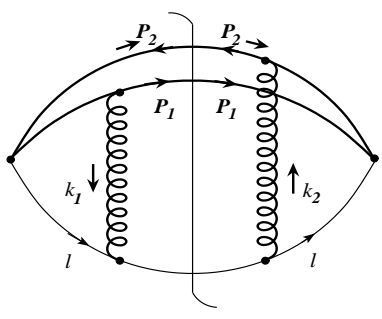

(I)

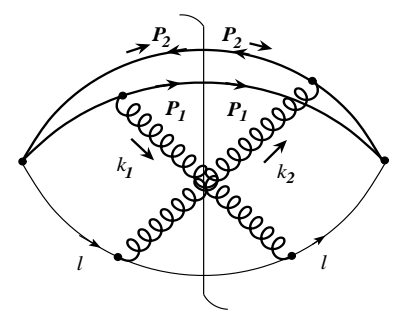

(II)

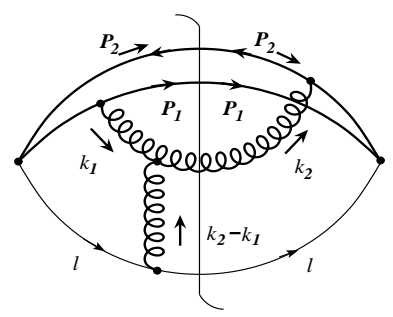

(III)

Fig. 4. Representative NNLO contributions to $g \rightarrow Q \bar{Q}$ fragmentation in eikonal approximation, see Ref. [4] for the details.

For quarkonium production, all heavy quark pairs with invariant mass less than a pair of open-flavor heavy mesons could become a bound quarkonium. Therefore, the effective velocity of heavy quark pair in the production could be much larger than that in decay. For charmonium production, charm quark velocity, $v_{c} \sim\left|\vec{q}_{c}\right| / m_{c} \leq \sqrt{\left(4 M_{D}^{2}-4 m_{c}^{2}\right) /\left(4 m_{c}^{2}\right)} \sim 0.88$, is not small, and therefore, the velocity expansion for charmonium production may not be a good approximation, unless one can identify and resum large contributions to all order in $v$ or have a factorized formalism at finite $v$. It was found in Ref. [14] that with the gauge-completed matrix elements, infrared singularities in the fragmentation function for a color octet pair to a singlet at NNLO are consistent with NRQCD factorization to all orders in $v$ or to a finite $v$,

$$
\mathcal{I}^{(8 \rightarrow 1)}(v)=\frac{\alpha_{s}^{2}}{4 \varepsilon}\left[1-\frac{1}{2 f(v)} \ln \left[\frac{1+f(v)}{1-f(v)}\right]\right]
$$

where $f(v)=2 v /\left(1+v^{2}\right)$. The result in finite $v$ is remarkably compact and intriguing, and should encourage further work on the factorization theorem.

\section{Summary and outlook}

Heavy quarkonium has two intrinsic scales and could be a good probe for the properties of the QGP or other dense medium. However, after more than 30 years since the discovery of $\mathrm{J} / \psi$, we still have not been able to fully understand the production mechanism of heavy quarkonium in high energy collisions, in particular, the transition from the heavy quark pair to a bound quarkonium. Although there are good reasons for each production model, none of the factorized production formalism, including that of the NRQCD model, has been proved theoretically. Further work on the factorization theorem is critical.

The transition from the pair to a bound meson is sensitive to the QCD confinement, and it is the dynamics of the transition that interacts and probes the properties of dense medium in heavy ion collisions. Nuclear matter could be an effective filter to distinguish the production mechanism [15]. Detailed study of 
nuclear dependence of heavy quarkonium production in hadron-nucleus collisions should provide invaluable information on the formation of heavy quarkonia in hadronic collisions. How good a probe the heavy quarkonium production

is completely depends on how well we understand and are able to calibrate the production.

\section{References}

[1] I. Arsene et al. [BRAHMS Collaboration], Nucl. Phys. A 757, 1 (2005); B. B. Back et al., Nucl. Phys. A 757, 28 (2005); J. Adams et al. [STAR Collaboration], Nucl. Phys. A 757, 102 (2005); K. Adcox et al. [PHENIX Collaboration], Nucl. Phys. A 757, 184 (2005).

[2] T. Matsui and H. Satz, Phys. Lett. B 178, 416 (1986).

[3] N. Brambilla et al. [Quarkonium Working Group], arXiv:hep-ph/0412158.

[4] G. C. Nayak, J. W. Qiu and G. Sterman, Phys. Lett. B 613, 45 (2005); Phys. Rev. D 72, 114012 (2005).

[5] J. C. Collins, D. E. Soper and G. Sterman, Nucl. Phys. B 263, 37 (1986).

[6] C. H. Chang, Nucl. Phys. B 172, 425 (1980); R. Baier and R. Ruckl, Phys. Lett. B 102, 364 (1981); E. L. Berger and D. L. Jones, Phys. Rev. D 23, 1521 (1981).

[7] E. Braaten, S. Fleming and T. C. Yuan, Ann. Rev. Nucl. Part. Sci. 46, 197 (1996); M. Kramer, Prog. Part. Nucl. Phys. 47, 141 (2001); G. T. Bodwin, Int. J. Mod. Phys. A 21, 785 (2006).

[8] F. Abe et al. [CDF Collaboration], Phys. Rev. Lett. 79, 572 (1997).

[9] H. Fritzsch, "Producing Heavy Quark Flavors In Hadronic Collisions: A Test Of Quantum Phys. Lett. B 67, 217 (1977); F. Halzen, Phys. Lett. B 69, 105 (1977); M. Gluck, J. F. Owens and E. Reya, Phys. Rev. D 17, 2324 (1978).

[10] J. F. Amundson, O. J. P. Eboli, E. M. Gregores and F. Halzen, Phys. Lett. B 390, 323 (1997).

[11] G. T. Bodwin, E. Braaten and G. P. Lepage, Phys. Rev. D 51, 1125 (1995) [Erratum-ibid. D 55, 5853 (1997)].

[12] A. A. Affolder et al. [CDF Collaboration], Phys. Rev. Lett. 85, 2886 (2000); CDF Collaboration, Notes 8212 and 8424 (06-06-22).

[13] J. P. Lansberg, Int. J. Mod. Phys. A 21, 3857 (2006) arXiv:hep-ph/0602091.

[14] G. C. Nayak, J. W. Qiu and G. Sterman, Phys. Rev. D 74, 074007 (2006).

[15] for example, see J. W. Qiu, J. P. Vary and X. f. Zhang, Phys. Rev. Lett. 88, 232301 (2002); D. Kharzeev and K. Tuchin, Nucl. Phys. A 770, 40 (2006); and references therein. 